Oriolus Revista Científica, ISSN: 2215-6100.1 (1). Julio, 2020, pp 14-26

Ordóñez Montoya, Arguedas Cortés

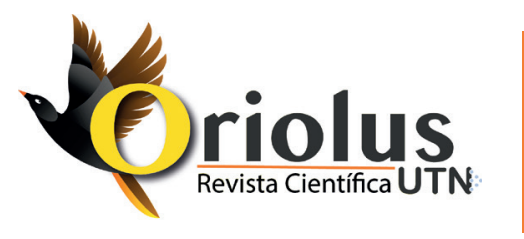

Oriolus Revista Científica

ISSN: 2215-6100

revista.cientifica.oriolus@utn.ac.cr

Universidad Técnica Nacional

\title{
Determinación del grado de bioalfabetización en estudiantes provenientes de escuelas cercanas a la zona de influencia de la Universidad Técnica Nacional
}

\section{Determination of the degree of bioliteracy in students from schools near to the area of influence of the National Technical University}

\author{
Elizabeth Ordóñez Montoya \\ Donald Arguedas Cortés \\ Determinación del grado de bioalfabetización en estudiantes provenientes de escuelas cercanas a \\ la zona de influencia de la Universidad Técnica Nacional \\ Oriolus Revista Científica, Vol.1, N. ${ }^{0} 1,2020$ \\ Universidad Técnica Nacional \\ Disponible en: http://revistas.utn.ac.cr/index.php/oriolus \\ Bibliografía: \\ Ordóñez Montoya, Elizabeth y Arguedas Cortés, Donald. "Determinación del grado de bioalfabeti- \\ zación en estudiantes provenientes de escuelas cercanas a la zona de influencia de la Universidad \\ Técnica Nacional". Revista Científica Oriolus 1, N. ${ }^{\circ} 1$ (2020): 14-26.
}


Oriolus Revista Científica, ISSN: 2215-6100.1 (1). Julio, 2020, pp 14-26

Ordóñez Montoya, Arguedas Cortés

\section{Determinación del grado de bioalfabetización en estudiantes provenientes de escuelas cercanas a la zona de influencia de la Universidad Técnica Nacional}

\section{Determination of the degree of bioliteracy in students from schools near to the area of influence of the National Technical University}

Elizabeth Ordóñez Montoya

Universidad Técnica Nacional, Costa Rica

eordonezm@utn.ac.cr

(iD https://orcid.org/0000-0002-2347-1601

Donald Arguedas Cortés

Universidad Técnica Nacional, Costa Rica

darguedas@utn.ac.cr

(iD) https://orcid.org/0000-0002-0127-4754
Recepción: 29 de abril del 2019

Aprobación: 11 de febrero del 2020

\section{Resumen}

En el presente estudio se determinó el grado de bioalfabetización en estudiantes provenientes de escuelas cercanas a la zona de influencia de la Universidad Técnica Nacional, donde participaron diez centros educativos procedentes de cuatro cantones en la provincia de Guanacaste, a saber: Cañas, Tilarán, Bagaces y Abangares. Un total de 141 estudiantes de tercero y cuarto año fueron abordados, el 50,35 \% correspondió al género femenino y 49,65\% al masculino. El grupo etario se mantuvo entre los 10 y 11 años de edad. Con la investigación se determinaron los principales factores que influyen sobre las comunidades en cuanto a temas ambientales. El mayor índice de bioalfabetización general $\left(I B_{L}\right)$ lo presentó la escuela San Antonio de Javilla en Cañas $(0,96)$ y el menor índice lo presentó la escuela Celestino Álvarez Ruíz de la comunidad de Montano de Bagaces. Únicamente dos escuelas (San Antonio de Javilla en Cañas y Agua Caliente de Bagaces) presentaron grados altos de bioalfabetización con valores de $I B_{L}$ iguales a 0,96 y 0,80 respectivamente.

Palabras claves: Bioalfabetización, índice, especies dulceacuícolas, escuelas, comunidades, educación.

\section{Abstract}

This article shows the obtained results from the research to determine the bioliteracy index degree in students from schools inside of the Universidad Técnica Nacional influence zone; it had ten educational centers participation coming from Cañas, Tilarán, Bagaces and Abangares. A total of 141 students were addressed, the $50.35 \%$ of the students were female gender and $49.65 \%$ were male. The age's average was among 10 and 11 years old. Upon completion of this investigation were obtained some results that show significant values to understand the communities' status studied in the environmental topic, for example: missing employment that produce as a bad result the poverty, hunting and fishing native species and others. The general highest bioliteracy index was shown by San Antonio de Javilla School in Cañas (0.96) and the lowest index was shown by Celestino Álvarez Ruíz school from Montano community in Bagaces. Only two educational centers (San Antonio de Javilla in Cañas and Agua Caliente in Bagaces) threw high bioliteracy degrees with $I B_{L}$ of 0.96 and 0.80 respectively.

Keywords: Bioliteracy, fishing, freshwater species, school, communities, children, education. 
Oriolus Revista Científica, ISSN: 2215-6100.1(1). Julio, 2020, pp 14-26

Ordóñez Montoya, Arguedas Cortés

\section{Introducción}

Al observar la cultura ambiental costarricense es visible la carencia de educación en este ámbito, se escucha hablar acerca de una "Costa Rica verde", pero el escenario no lo demuestra; Costa Rica vive una paradoja: tiene protegida la cuarta parte de su superficie y la mitad bajo cobertura forestal, pero mantiene a la vez una huella ecológica negativa y una alta conflictividad ambiental. Esta contradicción solo puede indicar la gravedad del uso insostenible que hace del resto del territorio, la persistencia de prácticas irresponsables y la débil gestión ambiental realizada (Programa Estado de la Nación, 2011). Es importante llevar a cabo una labor de alfabetización ambiental, orientada tanto a los jóvenes, como a los adultos enfocando mayor atención a sectores de la población en estado vulnerable, para fortalecer las bases de la opinión pública, inspirada en la responsabilidad que concierne a la protección y mejoramiento del medio (Sepúlveda \& Agudelo, 2012).

La conservación de la biodiversidad está determinada por valores y actitudes, y en la medida en que estos sean modificados con miras a un mejor entendimiento entre el ser humano y la naturaleza de la cual es parte, la riqueza natural del planeta tendrá mayores oportunidades de permanecer para el bien de las generaciones actuales y futuras (Arguedas, 2010). Según señala la Ley de Biodiversidad N. 7788 de 1998 en el capítulo VI, artículo 86: "La educación biológica deberá ser integrada dentro de los planes educativos en todos los niveles previstos, para lograr la comprensión del valor de la biodiversidad y del modo en que desempeña un papel en la vida y aspiración de cada ser humano".

El conocimiento vivencial y fortalecedor de los diversos tipos de competencias educativas circunscritas a los campos de conocimiento y disciplinas de las ciencias experimentales se llama bioalfabetización, un proceso que permite a los alumnos no solo complementar temas específicos impartidos en el aula, sino además construir procesos mentales y trascender a prácticas de gran utilidad actual o futura, también se fortalecen las capacidades cognitivas, ya que al ser la bioalfabetización un proceso vivencial es necesario ejecutar, ejercitar y asimilar estas capacidades (Museo Vivo, 2011).

En los últimos años, el Estado costarricense ha impulsado la educación ambiental, la que fue declarada de interés público y obligatoria en todo el país, tanto para escuelas públicas como privadas, mediante el Decreto N. ${ }^{\circ} 7235$ del 10 de junio de 1991. Su fortalecimiento y promoción se contemplan además en la Ley Orgánica del Ambiente (N. 7554 de 1995) y en la Ley de Biodiversidad (N. 7788 de 1998) (Muñoz, 2006). Debido a lo anterior, en el Área de Investigación de la Universidad Técnica Nacional (UTN), Sede Guanacaste, se implementó el programa de bioalfabetización, el cual, como señala el INBio (1999), permite al individuo valorar la biodiversidad y asumir su responsabilidad en el manejo y conservación. Es por ello que se pretende determinar la condición promedio de bioalfabetización de estudiantes provenientes de escuelas del área de influencia de la UTN, con el fin de desarrollar iniciativas para la Bioalfabetización y Biosensibilización en materia de uso, manejo y conservación de la biodiversidad acuática de Guanacaste.

\section{Materiales y métodos}

Área de estudio. Para la investigación se seleccionaron diez escuelas ubicadas dentro del área de influencia de la UTN (Región Chorotega), a saber: 1. Escuela Peñas Blancas, Colorado (10¹ 1 '43' 'N,

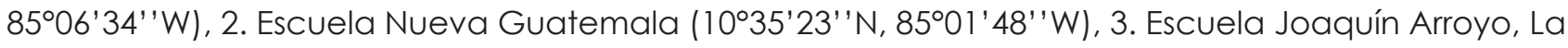

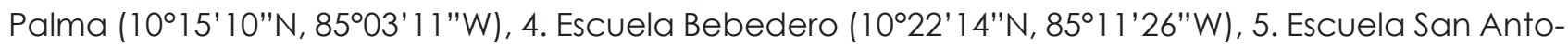

Esta obra esta bajo una Licencia de Creative Commons Atribución NoComercial-SinDerivar Internacional 
Oriolus Revista Científica, ISSN: 2215-6100.1 (1). Julio, 2020, pp 14-26

Ordóñez Montoya, Arguedas Cortés

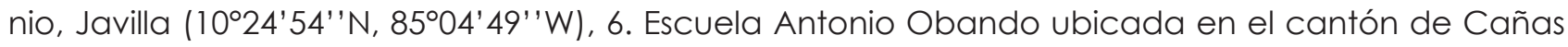

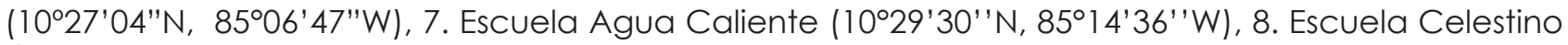

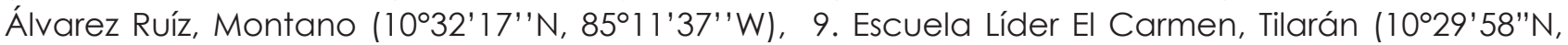
$84^{\circ} 54^{\prime} 26^{\prime \prime} \mathrm{W}$ ) y 10. Escuela Río Naranjo, Bagaces (10³9'02' 'N, 8505'50' 'W).

Toma de datos. Se realizó un muestreo no probabilístico intencional con 10 escuelas ( $\mathrm{n}=10$ ), para un total de 141 estudiantes distribuidos de la siguiente manera: 71 niñas y 70 niños provenientes del área de influencia de la Universidad Técnica Nacional, Sede Guanacaste; como referencia se tomó de perímetro las localidades delimitadas por el Área de Conservación Arenal Tempisque (Canje por bosques - FUNDACA, 2017) (ver figura. 1). De abril hasta noviembre 2017, se promovió la visita de los estudiantes al Laboratorio para Aguas y Reproducción de Especies Dulceacuícolas, dentro de las actividades realizadas se encuentran los recorridos por los tanques experimentales, donde se biosensibilizaron mediante una experiencia vivencial a través del contacto con especies acuícolas como el guapote lagunero (Parachromis dovii), langosta australiana (Cherax cuadricarinatus), almeja gigante (Anodontites Iuteola), tilapia gris (Oreochromis niloticus), tilapia roja (Oreochromis sp.), tilapia azul (Oreochromis aureus), caracol manzana (Pomacea flagellata), catfish (Ictalurus punctatus), posteriormente, se les impartió una pequeña presentación general sobre temas asociados a ecosistemas dulceacuícolas, además participaron en actividades, por ejemplo: colorearon dibujos de las especies vistas en el recorrido, resolver palabras ocultas del tema expuesto, encontrar en la "sopa de letras" términos relacionados con los ecosistemas dulceacuícolas y lo que se desarrolla en LARED; en seguida los estudiantes fueron llevados al laboratorio de calidad de aguas en donde tuvieron la oportunidad de conocer los diferentes equipos utilizados en los procesamientos de muestras, además de observar a través del microscopio; al finalizar, se les aplicó un instrumento de investigación (lista de cotejo), el cual contenía once preguntas referentes a la bioalfabetización.

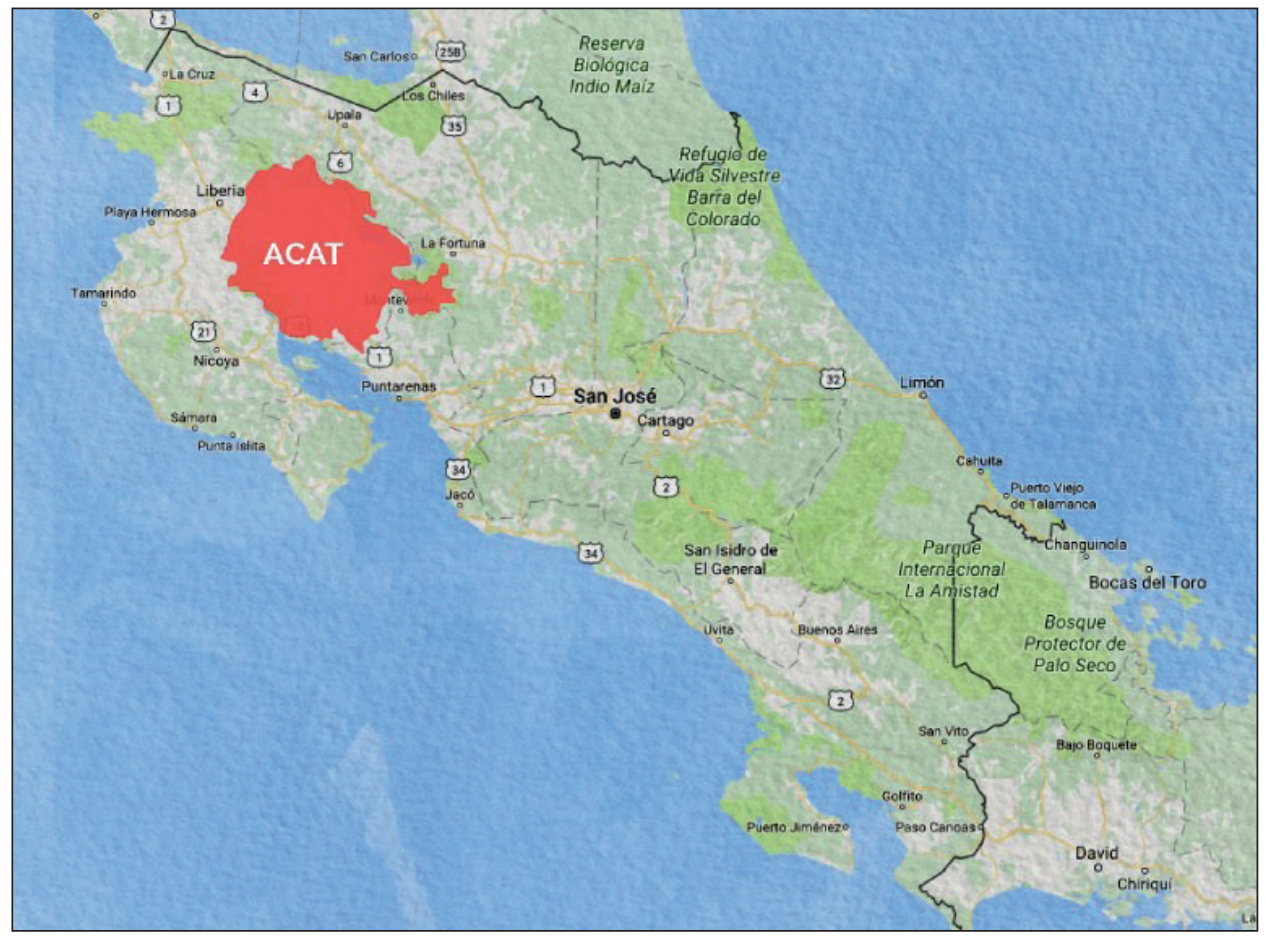

Figura 1. Perímetro Área de Conservación Arenal- Tempisque (ACAT). Fuente: Canje por bosques - FUNDACA, 2017. 


\section{Oriolus Revista Científica, ISSN: 2215-6100.1(1). Julio, 2020, pp 14-26}

Ordóñez Montoya, Arguedas Cortés

Estimación del Índice de Bioalfabetización. Para el cálculo de Índice de Bioalfabetización ( $\left[B_{L}\right)$ se elaboró una ecuación matemática que sintetiza el efecto de cuatro variables: 1. Visita al bosque, 3. Concepto Bioalfabetización, 8. Peligro de extinción-Guapote, 11. Pesca-Guapote, utilizadas como interrogantes en el instrumento aplicado. A cada una de las variables se les asignó un peso específico de acuerdo con el nivel de importancia dada a cada una de ellas. La ecuación es la siguiente:

$$
I B_{L}=\left[\frac{2}{3}(P \propto)+\frac{1}{3}(P \beta)+\frac{5}{1000}(P \gamma)+\frac{5}{1000}(P \varepsilon)\right]
$$

Donde:

$P \propto=$ la probabilidad de ocurrencia de respuestas negativas a la pregunta número 3 del instrumento por parte de los estudiantes / total de estudiantes entrevistados.

$\mathrm{P} \beta=$ la probabilidad de ocurrencia de respuestas positivas a la pregunta número 11 del instrumento por parte de los estudiantes / total de estudiantes entrevistados.

Py $=$ la probabilidad de ocurrencia de respuestas negativas a la pregunta número 8 del instrumento por parte de los estudiantes / total de estudiantes entrevistados.

$\mathrm{P} \varepsilon=$ La probabilidad de ocurrencia de respuestas negativas a la pregunta número 1 del instrumento por parte de los estudiantes / total de estudiantes entrevistados.

Escala para la clasificación de los valores probables del ( $\left(\boldsymbol{B}_{\boldsymbol{L}}\right)$. Para la distribución de los valores del grado de bioalfabetización se diseñó la tabla 1 de clasificación que contiene cuatro categorías probables aplicables a niños y niñas: I. Alto, II. Medio alto, III. Medio bajo y IV. Bajo.

Tabla 1. Clasificación del grado de bioalfabetización según el Índice de Bioalfabetización $(\underline{\underline{I}} \underline{L})$ mediante escala de colores.

\begin{tabular}{|ccc|}
\hline Grado de Bioalfabetización & $\left(\boldsymbol{I} \boldsymbol{B}_{\boldsymbol{L}}\right)$ & Escala de colores $\left(\boldsymbol{I} \boldsymbol{B}_{\boldsymbol{L}}\right)$ \\
\hline Alto & $0,75-1$ & \\
Medio alto & $0,50-0,75$ & \\
Medio bajo & $0,25-0,50$ & \\
Bajo & $0-0,25$ & \\
\hline
\end{tabular}

Fuente: elaboración propia, 2017. 
Oriolus Revista Científica, ISSN: 2215-6100.1 (1). Julio, 2020, pp 14-26

Ordóñez Montoya, Arguedas Cortés

\section{Resultados}

Índice de bioalfabetización: En la tabla 1 se muestra el rango de los valores de los Índices de Bioalfabetización $\left(I B_{L}\right)$ para cada una de las escuelas presentes en este estudio. Un total de 141 estudiantes de tercero y cuarto año distribuidos en diez escuelas provenientes de las zonas de influencia de la Universidad Técnica Nacional, Sede Guanacaste, fueron abordados. El 50,35 \% de los estudiantes del género femenino y 49,65\% del masculino. El grupo etario se mantuvo entre los 10 y 11 años de edad. El mayor Índice de Bioalfabetización general $\left(I B_{L}\right)$ lo presentó la escuela San Antonio de Javilla de Cañas (0.96). Únicamente dos escuelas (San Antonio de Javilla en Cañas y Agua Caliente de Bagaces) presentaron grados altos de bioalfabetización con valores de $I B_{L}$ iguales a 0,96 y 0,80, respectivamente. Tres escuelas (Río Naranjo de Bagaces, Nueva Guatemala en Cañas y Peñas Blancas de Abangares) presentaron grados medios altos de bioalfabetización: 0,50, 0,59 y 0,73, respectivamente (ver tabla 1). La escuela de Bebedero de Cañas ( $I B_{L}=0,32$, Joaquín Arroyo de la Palma de Abangares ( $\left(I B_{L}=0,34\right)$, la escuela Antonio Obando de Cañas con $\left(I B_{L}=0,40\right)$ y la de El Carmen de Tilarán $\left(\underline{\left.I B_{L}=0,49\right)}\right.$ se ubicaron dentro del grado medio bajo de bioalfabetización. El menor fue mostrado por la escuela Celestino Álvarez Ruíz de la comunidad de Montano de Bagaces, con un valor de 0,25.

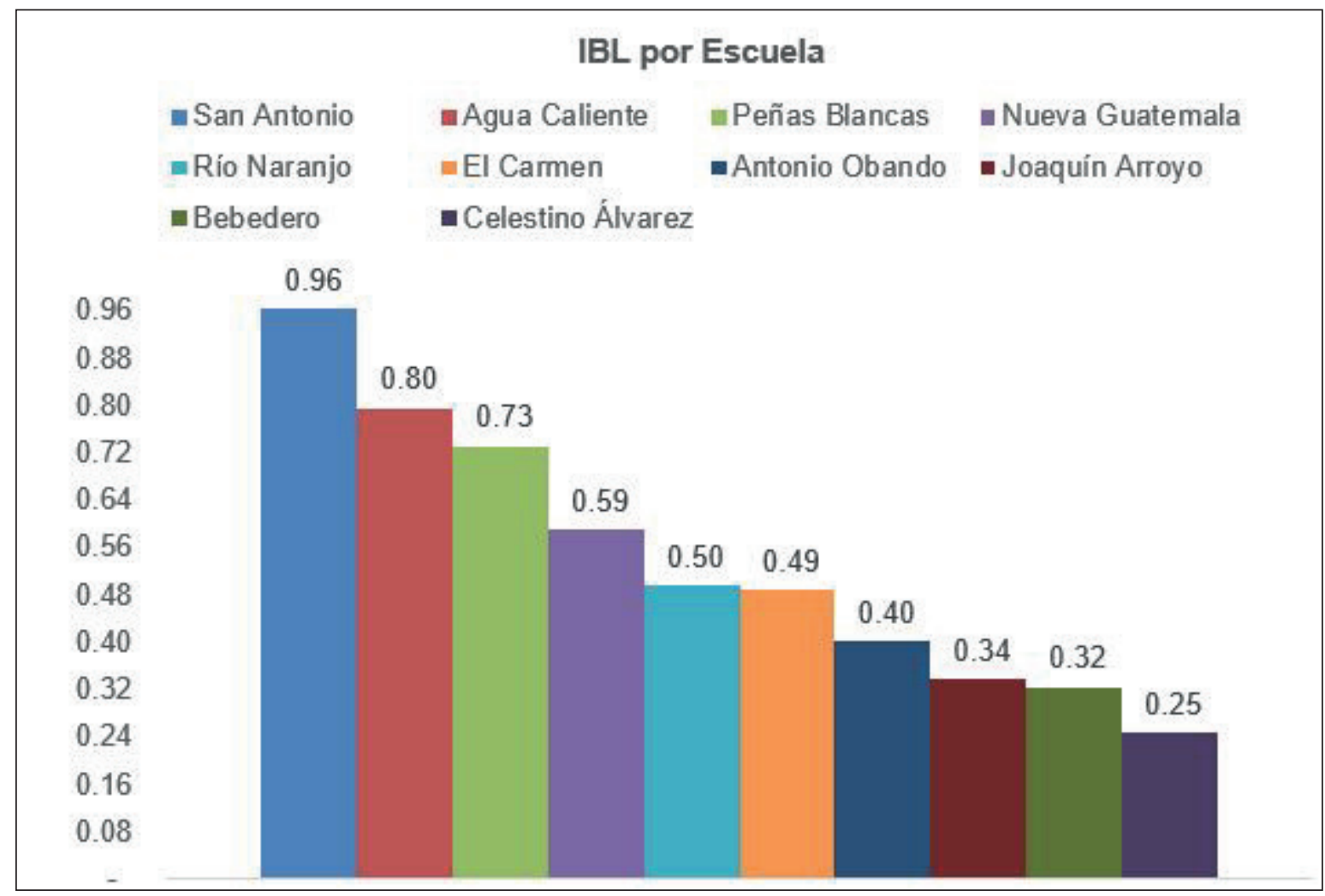

Figura 2. Índice de Bioalfabetización por centro educativo. Fuente: elaboración propia, 2017.

Índice de Bioalfabetización en las escuelas del cantón de Cañas: Aunque los valores de $I B_{L} s$ son diferentes entre géneros cuando se comparan las escuela de San Antonio $I B_{L} f$ (niñas) = 1,0 e $I B_{L} m$ (niños) = 0,94 con Bebedero $\underline{I B_{L} f}=0,34 \mathrm{e} \underline{I B_{L} m}=0,31$; no se encontraron diferencias significativas $(\mathrm{X} 2$ 
Oriolus Revista Científica, ISSN: 2215-6100.1(1). Julio, 2020, pp 14-26

Ordóñez Montoya, Arguedas Cortés

$=0,01, p>0,05)$ por lo que los $I B_{L}$ no dependen del género en este caso. De igual manera, cuando se comparó a la escuela de San Antonio $\mid I B_{L} f=(1,0)$ y niños $(0,94)$ con Antonio Obando $\left\lfloor B_{L}\right\rfloor=$ 0,40 y niños $=0,40$, a pesar de que el $I B_{L}$ de la primera fue superior tanto en niños como en niñas, no se presentaron diferencias significativas $(x 2=0,05, p>0,05)$. En lo que respecta a la esquela de San Antonio y Nueva Guatemala se encontró que los $I B_{L} f$ fueron de 1,0, mientras que los $I B_{L} m$ en San Antonio se mantuvieron mayor que en Nueva Guatemala $I B_{L} f=0,01$ de tal manera que existe una diferencia altamente significativa $(x 2=48,3, p<0,001)$.

Las niñas de la escuela de Bebedero poseen un $I B_{L} f$ de 0,34 y las de la escuela de Antonio Obando de 0,40. Al comparar los índices masculinos para ambas escuelas, la primera presentó un índice menor: $I B_{L} m$ igual a 0.31 y la segunda, 0,40, sin encontrar diferencias significativas $(x 2=0,05, p>$ $0,05)$. Entre la escuela de Antonio Obando y la escuela de Nueva Guatemala se presentaron diferencias significativas ( $2=39,9, \mathrm{p}<0,001)$; por lo que el el $I B_{L} f$ de Nueva Guatemala es mayor 1,0, pero inferior en masculino $\left(I B_{L} m=0,01\right)$. El $I B_{L} n$ fue mayor en la escuela de Nueva Guatemala (1) al obtenido por la escuela de Bebedero $(0,34)$ (ver figura 3), sin embargo un $I B_{L} m$ inferior 0,01 y 0,31, respectivamente $(x 2=33,9, p<0,001)$.

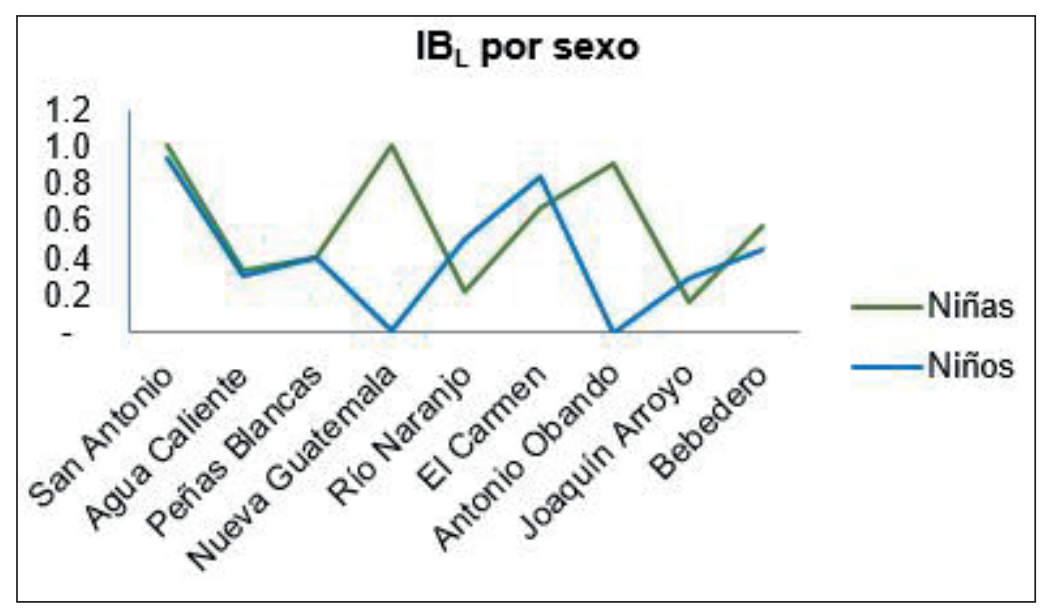

Figura 3. Índice de Bioalfabetización de LARED según sexo por escuelas. Fuente: elaboración propia, 2017.

Índice de Bioalfabetización escuelas del cantón de Abangares. De las dos escuelas pertenecientes al cantón de Abangares, Peñas Blancas presentó un $I B_{L}=0,73$ en contra posición con el mostrado por Joaquín Arroyo $\left(I B_{L}=0,34\right)$. En cuanto al género Peñas Blancas presenta un $I B_{L} f$ de 0,68 el $I B_{L} m$ de 0,84 , mientras que Joaquín Arroyo presentó $I B_{L} f$ de 0,23 y $I B_{L} m$ de 0,50, no existieron diferencias significativas entre los valores de $I B_{L}$ entre escuelas según género ( $\left.x 2=35,8, p>0,05\right)$.

Índice de Bioalfabetización escuelas del cantón de Bagaces. Tres escuelas fueron analizadas pertenecientes al cantón de Bagaces. Existieron diferencias altamente significativas cuando se compararon los valores de $I B_{L} f$ e $I B_{L} m$ según género entre las escuelas de Agua Caliente y Celestino Álvarez (X2 $=72,7, p<0,001)$. De igual manera, al comparar Agua Caliente con la escuela de Río Naranjo $I B_{L} f=0,91$ e $I B_{L} f=0,58$ y con valores de $I B_{L} m=0$ e $I B_{L} m=0,45$, se encontró presencia de una relación directa entre los valores de $I B_{L}$ según el género entre escuelas $(X 2=51,7, p<0,001)$. Esta significancia estadística se encontró cuando se compararon las escuelas de Celestino Álvarez y Río 
Oriolus Revista Científica, ISSN: 2215-6100.1(1). Julio, 2020, pp 14-26

Ordóñez Montoya, Arguedas Cortés

Naranjo (x2 = 4,76, $\mathrm{p}<0,05)$ aunque la primera mostró un $I B_{L} f$ inferior que la segunda al igual que el valor obtenido para el $I B_{L} m$.

Índice de Bioalfabetización escuela del cantón de Tilarán. Solamente una escuela (El Carmen) proveniente del cantón de la altura fue considerada en esta investigación. Aquí se brindó un valor de $I B_{L}$ de 0,49 (grado medio bajo de bioalfabetización). Se puede rescatar que esta escuela presentó un $I B_{L} f=0,40$ y los niños un $I B_{L} m$ de 0,58 .

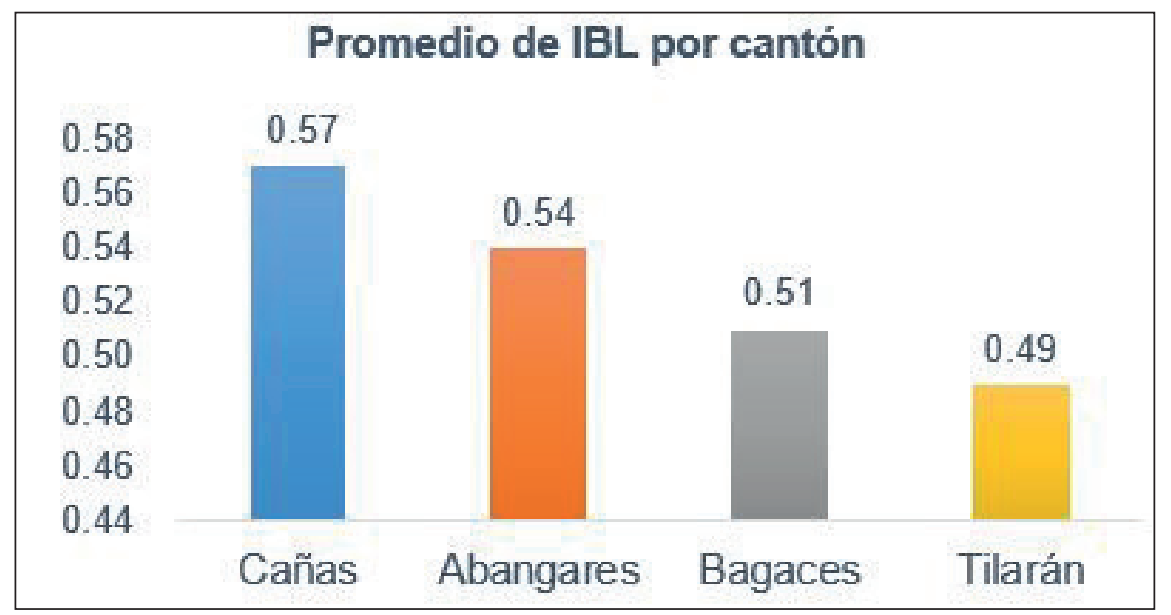

Figura 4. Promedio de Índice de Bioalfabetización ( $\left(I B_{L}\right)$ por cantón. Fuente: elaboración propia, 2017.

Tabla 2. Valores del índice de bioalfabetización $\left(I B_{L}\right)$ por centros educativos según el género.

\begin{tabular}{|c|c|c|c|c|c|c|c|c|c|c|c|}
\hline Escuela & Cantón & Niñas & Niños & $\begin{array}{c}\text { Número } \\
\text { Estudiantes }\end{array}$ & $\mathrm{IB}_{\mathrm{L}}$ & Escala & Niñas & Escala & Niños & Escala & $\begin{array}{c}B_{L} \\
\text { Promedio }\end{array}$ \\
\hline San Antonio & Cañas & 3 & 5 & 8 & 0,96 & A & 1,0 & A & 0,94 & A & 0,97 \\
\hline Bebedero & Cañas & 13 & 10 & 23 & 0,32 & MB & 0,34 & MB & 0,31 & MB & 0,32 \\
\hline Antonio Obando & Cañas & 11 & 11 & 22 & 0,40 & MB & 0,40 & MB & 0,40 & MB & 0,40 \\
\hline Nueva Guatemala & Cañas & 7 & 5 & 12 & 0,59 & MA & 1,0 & A & 0,01 & B & 0,51 \\
\hline Joaquín Arroyo & Abangares & 6 & 4 & 10 & 0,34 & MB & 0,23 & B & 0,50 & MA & 0,37 \\
\hline Peñas Blancas & Abangares & 2 & 4 & 6 & 0,73 & MA & 0,68 & MA & 0,84 & A & 0,76 \\
\hline Agua Caliente & Bagaces & 7 & 1 & 8 & 0,80 & A & 0,91 & A & $-^{*}$ & B & 0,45 \\
\hline Celestino Álvarez & Bagaces & 4 & 7 & 11 & 0,25 & B & 0,17 & B & 0,29 & MB & 0,23 \\
\hline Río Naranjo & Bagaces & 7 & 12 & 19 & 0,50 & MA & 0,58 & MA & 0,45 & MB & 0,51 \\
\hline El Carmen & Tilarán & 11 & 11 & 22 & 0,49 & MB & 0,40 & MB & 0,58 & MA & 0,49 \\
\hline Total & & 71 & 70 & 141 & & & & & & & \\
\hline Porcentaje & & $50,35 \%$ & $49,65 \%$ & & & & & & & & \\
\hline
\end{tabular}

*Solamente participó un niño, el cual no respondió. Fuente: elaboración propia, 2017. 
Oriolus Revista Científica, ISSN: 2215-6100.1(1). Julio, 2020, pp 14-26

Ordóñez Montoya, Arguedas Cortés

\section{Discusión}

Esta es la primera investigación que se realiza para determinar el grado de bioalfabetización en estudiantes de escuelas de la zona de influencia de la Universidad Técnica Nacional, Sede Guanacaste, y quizás el primer estudio en Costa Rica que determina la estimación para un Índice de Bioalfabetización.

El promedio poblacional de los cantones donde se localizan los centros educativos en el estudio es de 5 648,5 mujeres y 5 814,2 hombres (Ruíz, 2010), lo cual representa el 49,28\% y 50,72 \%, respectivamente, similar distribución porcentual para los géneros de los estudiantes muestreados en esta investigación.

Índice de Bioalfabetización escuelas del cantón de Cañas: no se encontró una relación directa entre los valores de $\overline{I B_{I}}$ en estudiantes según el género al compararse las escuelas San Antonio y Bebedero. Una posible explicación a lo que sucede con la condición de género de la escuela San Antonio es que está positivamente influenciado por la naturaleza que rodea la comunidad de Javilla, por lo tanto, niñas y niños valoran la biodiversidad y pareciera ser que la diferencia de clase social en los estudiantes de esta comunidad no determina que la pobreza resulte en deterioro a la flora y fauna silvestre como pudiese estar ocurriendo en otras comunidades de otros cantones aledaños a la Sede Guanacaste de la Universidad Técnica Nacional.

La comparación de los $\overline{I B_{I}}$. para los géneros en las escuelas de San Antonio y Antonio Obando no mostraron diferencias significativas $(x 2=0,05, p>0,05)$, este resultado puede ser debido a que la condición social existente en el barrio San Martín determina, de igual manera, a niñas y niños, en otras palabras, es indiferente ser una niña o un niño de tercero y cuarto grado cuando se habla en términos de Índice de Bioalfabetización, el ambiente negativo, socialmente hablando que influencia a este caserío, influye de igual manera al género de estudiantes de tercero y cuarto grado.

Para la escuela de San Antonio y Nueva Guatemala los índices de bioalfabetización presentaron diferencia altamente significativa $(X 2=48,3, p<0,001)$ según la variable género, esto es mostrado por el valor brindado para el Índice de Bioalfabetización para niños de la escuela de Nueva Guatemala ( $I B_{L} m=0,01$ ) explicado por causa de que existe en el pueblo el hábito de que los niños acompañan a los padres a actividades de pesca y cacería ilegal. Aspecto que no se presentas en las niñas, quienes poseen un Índice de Bioalfabetización de categoría alta ( $\operatorname{IB} L=1,0$ ), por lo que el tiempo libre de las niñas de tercero y cuarto grado de la Escuela Nueva Guatemala lo invierten en labores domésticas acompañando a sus madres. Esta información fue debidamente constatada en la aplicación del instrumento.

Al compararse los $\overline{I B_{I}}$ de las escuelas de Bebedero y Antonio Obando según el género no mostraron diferencias significativas ( $x 2=0,05, p>0,05)$, posiblemente relacionada con la realidad sociocultural y económica que muestran estos lugares, aunado al ambiente donde están creciendo/educando por igual los niños y las niñas. Nuevamente, es indiferente ser una niña o un niño de la población en estudio cuando se habla en términos de Índice de Bioalfabetización.

La diferencia significativa $(x 2=39,9, \mathrm{p}<0,001)$ encontrada entre los centros educativos de Antonio Obando y Nueva Guatemala son explicadas por el factor de asociación o nicho que existe entre el padre de familia y el niño en los hogares del pueblo de Nueva Guatemala tal y como se describió anteriormente, lo que se evidenció en el instrumento aplicado.

Esta obra esta bajo una Licencia de Creative Commons Atribución NoComercial-SinDerivar Internacional 
Oriolus Revista Científica, ISSN: 2215-6100.1(1). Julio, 2020, pp 14-26

Ordóñez Montoya, Arguedas Cortés

La situación inédita comentada para el $\overline{\mathrm{IB}_{\mathrm{L}} \mathrm{m}}$ de la escuela de Nueva Guatemala determinó la existencia de una diferencia altamente significativa $(x 2=33,9, p<0,001)$ al compararla con Bebedero, la cual presenta una socio economía poco desarrollada.

Índice de Bioalfabetización escuelas del cantón de Abangares: las dos escuelas pertenecientes al cantón de Abangares con respecto a los $I B_{L} f$ e $I B_{L} m$ encontrados, presentaron relación X2 = 35,8, p $>0,05$, probablemente apoyada por la similitud funcional de la pesca artesanal y generacional que se tiene por costumbre tanto en la comunidad de La Palma como en Colorado.

Índice de Bioalfabetización escuelas del cantón de Bagaces: la ausencia de relación (X2=72.7, $\mathrm{p}<0.001)$ para los valores de $I B_{L}$ obtenidos según género cuando se compararon las escuelas de Agua Caliente con Celestino Álvarez y Agua Caliente con Río Naranjo ( $x 2=51,7, p<0,001)$ tiene su explicación en que el único niño de la escuela Agua Caliente que visitó LARED no completó el instrumento aplicado, lo que representa la diferencia altamente significativa encontrada. La posible causa de que una niña y un niño de la escuela de Río Naranjo presente diferencias significativas al analizar el género $(x 2=4,76, p<0,05)$ es porque las familias del caserío de Montano de Bagaces frecuentan los reservorios (ríos, quebradas, pozas) aledaños con la finalidad de realizar la pesca furtiva, en contra posición, con los pobladores de la comunidad de Río Naranjo, quienes tienen costumbres similares a los vecinos del pueblo de Nueva Guatemala.

Índice de Bioalfabetización escuela del cantón de Tilarán: por ser la única escuela ubicada en el cantón de Tilarán no se generó análisis estadístico inferencial. Este cantón quedará para estudios posteriores. Asimismo, por la naturaleza de la presente investigación (prácticamente inédita) los resultados no se pueden discutir ni comparar con estudios similares.

El presente estudio es un esfuerzo de investigación sobre el tema de bioalfabetización que trabaja de manera conjunta lo experimental, étnico, biológico, ambiental y pedagógico, con la finalidad de generar información que caracterice en primera instancia la población educativa primaria de tercero y cuarto grado y en segunda instancia los primeros trazos de los perfiles socio biológicos de ciertas comunidades aledañas a la Sede Guanacaste de la Universidad Técnica Nacional. No existen estudios previos similares al desarrollado. Algunos esfuerzos en el campo de la gestión de la bioalfabetización, pero no en la línea de la investigación científica han sido desarrollados por diferentes grupos en México y el INBio en Costa Rica.

Índice de bioalfabetización: el resultado elevado del $I B_{L}$ obtenido por la escuela San Antonio de Javilla puede asociarse con que este centro educativo presenta la modalidad de escuela unidocente, de acuerdo con (MEP, 2010) en esta clase de escuelas los estudiantes comparten como una familia y la mayoría viajan por caminos rodeados de naturaleza, animales y, algunas veces, atraviesan quebradas rodeadas de bosque, anatomía ecológica similar donde se encuentra inmiscuido dicho centro. TIC (2007) reporta que este tipo de estudiantes tienen que esforzarse más por asistir a las lecciones, lo que podría ser ventaja para que valoren las experiencias vivenciales circundantes a su tiempo lectivo si se comparan con una escuela superior o igual al tipo D2 (clasificación de escuelas) (MEP, 2010). Además, resulta para el maestro, la fácil difusión del conocimiento dentro del aula en temas relacionados con la interiorización al respeto que se debe fomentar hacia la naturaleza. Además, resulta interesante de que a pesar que la escuela San Antonio de Javilla se localiza a poca distancia del centro de población del cantón de Cañas, esta comunidad no se encuentra tan influenciada por la problemática social y económica que se vivencia en el centro de Cañas. A pesar

Esta obra esta bajo una Licencia de Creative Commons Atribución NoComercial-SinDerivar Internacional 
Oriolus Revista Científica, ISSN: 2215-6100.1(1). Julio, 2020, pp 14-26

Ordóñez Montoya, Arguedas Cortés

de que hijos e hijas de jornaleros se matriculan en la escuela de Javilla, igualmente lo hacen los de dueños de medias y grandes extensiones de fincas ganaderas con un mayor poder adquisitivo. Condición similar social-económica muestran los estudiantes de la escuela de Agua Caliente, Bagaces y su alto valor de $I B_{L}$ podría deberse a lo discutido, anteriormente, para la escuela de San Antonio. Sin embargo, el factor que estaría determinando directamente el $\overline{I B_{L}}$ es lo citado por TIC (2007). Las escuelas de Río Naranjo de Bagaces, Nueva Guatemala en Cañas y Peñas Blancas de Abangares se encuentran ubicadas en perímetros rurales según el IDHC -PNUD (2011), pero con condiciones distintas, económicamente hablando. La similitud de los valores para los $I B_{L}$ brindado puede estar determinado en que tanto la primera, como la segunda escuela se encuentran localizadas en zonas cubiertas por bosques y montañas y la subsistencia económica se fundamenta en la elaboración de productos derivados de la leche y provenientes de la agricultura, lo cual genera una sensibilización hacia la naturaleza. No obstante, esta ventaja paisajista podría transformarse en una desventaja para los niños y niñas que inician su proceso de educación primaria en estas escuelas; la tercera escuela presenta condiciones de desarrollo rural similar a las dos anteriores, únicamente que su economía depende directamente en gran medida de los productos derivados de la pesca, quizás los niños de esta escuela en sus primeros años podrían estar desarrollando respeto hacia la biodiversidad acuática, reflejado en el $\overline{I B_{L}}$ más alto de estos tres centros educativos, por lo que tener una experiencia con peces en el LARED produzca un feedback experience (sentir de nuevo la experiencia vivida). Este importante valor podría variar cuando estos dicentes alcancen una mayor edad y formen núcleos familiares en su comunidad. Los resultados muestran que de las cuatro escuelas clasificadas como medio bajo Bebedero posee un valor para $I B_{L}$ menor con respecto a las restantes. Posiblemente se deba a la relación de pobreza en que vive esta comunidad y que afecta directamente la bioeducación de los estudiantes. Su economía se fundamenta en la corta de caña, actividad que retribuye pocos ingresos familiares, madres solteras y gran cantidad de emigrantes nicaragüenses. Aunque este distrito limita con áreas silvestres protegidas (ASP) como el Parque Nacional Palo Verde, la Reserva Forestal Taboga y Reserva Biológica Lomas de Barbudal, este factor no ha sido positivo para la sensibilización hacia la naturaleza y, en contraste, ha prevalecido más aspectos asociados a la pobreza, por ejemplo: la pesca ilegal y la cacería furtiva. Falta una mayor incidencia por parte del Sistema Nacional de Áreas de Conservación y del Área de Conservación Arenal Tempisque. Con la escuela Joaquín Arroyo del poblado de La Palma el valor del $I B_{L}$ podría explicarse que está determinado por la misma condición presentada por la escuela de Peñas Blancas, cuando visitan LARED presencian un feedback experience de tal manera que ambos centros educativos concuerdan primero en ubicación geográfica cantonal y segundo en una cultura de pesca artesanal que para estos casos resulta positiva para los estudiantes de tercero y cuarto grado. A pesar de que la escuela Antonio Obando se ubica en el cantón de Cañas al igual que el centro educativo que posee el mayor $I B_{L}$ reportado en este estudio, parece que una de las condiciones determinantes para que exista variación entre los valores de índices sería la comunidad/barrio donde se encuentra ubicado dicho centro. Según el Índice de Desarrollo Humano Cantonal del Programa de las Naciones Unidas para el Desarrollo (2011) el barrio San Martín es una zona que presenta una gran problemática de vicios, drogas, desempleo, poca oferta de trabajo, no hay naturaleza alrededor; lo que repercute negativamente el valor cuando se determina el grado de bioalfabetización. De la misma manera, la escuela del Carmen que, también, se ubica en un grado medio bajo de bioalfabetización, pero esta vez en el cantón de Tilarán con un Índice de Pobreza Humana Cantonal (IPHC) de 19,0\% en contraposición al cantón de Cañas de 18,8 \% (PNUD, 2011); no obstante, también es un barrio con problemas de índoles sociales y económicos, pero con mayor porcentaje de paisaje y naturaleza que la escuela Antonio Obando. El valor bajo de la escuela Celestino Álvarez Ruíz de la comunidad de Montano de Bagaces ( $I B_{L}=0.25$ ) podría estar determinado por la carencia de fuentes de em-

Esta obra esta bajo una Licencia de Creative Commons Atribución NoComercial-SinDerivar Internacional 
Oriolus Revista Científica, ISSN: 2215-6100.1(1). Julio, 2020, pp 14-26

Ordóñez Montoya, Arguedas Cortés

pleo en el caserío y por la cultura de caza furtiva cotidiana de los habitantes, además de la pesca ilegal, a pesar de que la tasa de alfabetización adulta es de 94,0 \% para el cantón de Bagaces, la población es muy longeva y apenas alcanza la primaria completa (PNUD, 2011). Estas situaciones hacen que la naturaleza (flora y fauna) que circunda este pueblo no permita crear sensibilización en niños y niñas de tercero y cuarto grado del centro educativo mencionado. Este es el único caso donde el factor naturaleza cumple una función inversamente proporcional al valor estimado de $I B_{L}$ en la escuela.

\section{Agradecimientos}

Deseamos expresar nuestro agradecimiento a los docentes y directores de los centros de enseñanza que participaron en esta investigación por el interés mostrado en facilitar y gestionar los respectivos permisos establecidos por el Ministerio de Educación Pública (MEP) para que los estudiantes visitaran el LARED-UTN. Este trabajo ha sido financiado por la Ley de Pesca y Acuicultura N. ${ }^{\circ} 8436$ y realizado con la colaboración de las Direcciones Regionales (Cañas, zona norte-norte Upala) y el Sistema Nacional de Áreas de Conservación (SINAC) representado por el Área de Conservación Arenal Tempisque (ACAT).

\section{Bibliografía}

Arguedas, D. Implementación de un programa de Bioalfabetización como apoyo alternativo al currículum impartido por la Universidad Técnica Nacional en la sede Guanacaste. Tesis para optar por el grado de licenciatura en educación con énfasis en docencia, UMCA, 2010.

Bioalfabetizando a tus alumnos, 2011. http://bioalfabetizacioncompetenciaeducativa.blogspot. com/2011/05/ante-la-enorme-responsabilidad-que-como.html

Canje por bosques - FUNDACA. Planes específicos de delimitación de ACAT, 2017. https://canjeporbosques.org/planes-especificos-de-delimitacion-de-acat/

INBio. Los esfuerzos de conservación de la biodiversidad, Educación y conciencia pública, 1999. http://www.inbio.ac.cr

Informe Estado de la Nación. Decimoséptimo estado de la nación, 2010. Pobreza, desigualdad en la distribución del ingreso y empleo en un contexto lenta recuperación económica y crisis fiscal. San José: Programa Estado de la Nación, 2011.

Informe Estado de la Nación. Sinopsis. Decimoséptimo Informe Estado de la Nación en Desarrollo Humano Sostenible. San José: Programa Estado de la Nación, 2011.

Ministerio de Educación Pública. Capítulo VIl: Dignificación y profesionalización del recurso humano, 2010. https://www.mep.go.cr/sites/default/files/page/adjuntos/memoria-mep-web-cap-vii. pdf

Muñoz, E. S. (2006). "La evolución de la educación ambiental en Costa Rica". Revista de Ciencias Sociales (Cr) 1, N. ${ }^{\circ} 111-112$ (2006): 714-260.

Programa de las Naciones Unidas para el Desarrollo. Atlas del Desarrollo Humano Cantonal de Costa Rica, 201 1. https://docplayer.es/8357689-Atlas-del-desarrollo-humano-cantonal-de-costa-rica-2011.html

Esta obra esta bajo una Licencia de Creative Commons Atribución NoComercial-SinDerivar Internacional 
Oriolus Revista Científica, ISSN: 2215-6100.1(1). Julio, 2020, pp 14-26

Ordóñez Montoya, Arguedas Cortés

Ruiz, C. Diagnóstico estratégico de la Región Chorotega. Presidencia Ejecutiva. Dirección de Planificación. Instituto Costarricense de Acueductos y Alcantarillados, 2010.

Sepúlveda, E., \& Agudelo, N. Thinking environmental education: historical approximation to international legislation from a critical perspective. Luna Azul, 35 (2012): 201-265.

TIC, P. Escuela unidocente y multigrado Honduras, 2007. https://issuu.com/educacionrural/docs/ escuelas_unidocentes_honduras_2007 